\title{
Los erizos Arbacia incisa y Eucidaris thouarsii (Echinodermata) como agentes de biocontrol del "fouling" en canastas de cultivo de Crassostrea gigas (Mollusca: Ostreidae)
}

\author{
Jorge Sonnenholzner ${ }^{1}$, Gema Alcivar $^{2}$, Adrián Marquez ${ }^{1} \&$ César Lodeiros ${ }^{1,2 *}$ \\ 1. Escuela Superior Politécnica del Litoral, ESPOL, Centro Nacional de Acuicultura e Investigaciones Marinas. 30.5, \\ Vía Perimetral, Guayaquil, Ecuador. sonnenhol@espol.edu.ec, ajmm16@hotmail.com, cesarlodeirosseijo@yahoo.es \\ 2. CALADEMAR S.A., San Pablo, Punta Blanca, Provincia de Santa Elena, Ecuador. biogealin@gmail.com \\ 3. Instituto Oceanográfico de Venezuela, Universidad de Oriente, Cumaná 6101, Venezuela. \\ * Correspondencia
}

Recibido 12-IX-2016. Corregido 19-III-2017. Aceptado 30-IV-2017.

\begin{abstract}
The sea urchins Arbacia incisa and Eucidaris thouarsii (Echinodermata) as fouling biocontrol agents in culture cages of Crassostrea gigas (Mollusca: Ostreidae). Fouling clogs cultivation cages of bivalves afecting their growth. We tested biocontrol with sea urchins in the suspended culture of $C$. gigas during one month. The experimental design included: 1) oysters without sea urchins, 2) oysters with the black sea urchin Arbacia incisa (50.5 $\pm 0.43 \mathrm{~mm}$ of diameter testa-DT), 3) oysters with the pencil sea urchin E. thouarsii (34. $2 \pm 2.13 \mathrm{~mm} \mathrm{DT}$ ) and 4) oysters without sea urchins with a pearl net protective bag (to avoid predation). The dry mass of the pearl nets and the oyster soft tissues were determined at the beginning and end of the experiment. There was similar fouling in cages without urchins $(55.3 \pm 5.80 \mathrm{~g})$ and with $\mathrm{A}$. incisa $(46.1 \pm 2.84$ g). These amounts were significantly higher than those found in cages with $E$. thouarsii (39.1 $\pm 1.9 \mathrm{~g})$ and with protective sacs without urchins $(35.6 \pm 5.17 \mathrm{~g})$. The growth of $C$. gigas with E. thouarsii was significantly higher $(0.49 \pm 0.089 \mathrm{~g})$. We recommend E. thouarsii as a fouling biocontrol agent for C. gigas. Rev. Biol. Trop. 65(Suppl. 1): S35-S41. Epub 2017 November 01.
\end{abstract}

Key words: pearl nets; oysters; biocontrol; marine aquaculture; polyculture.

La adherencia de material inerte y organismos vivos fijados a estructuras en el mar (fouling) afectan el crecimiento y la supervivencia de moluscos bivalvos en condiciones de cultivo (Uribe, Lodeiros, Felix-Pico \& Etcherepare, 2001; Fitridge, Dempster, Guenther \& De Nys, 2012; Lacoste \& Gaertner-Mazouni, 2014), lo cual genera altos niveles de mantenimiento, llegando a suponer hasta el 30\% de los costos operacionales (Lacoste \& GaertnerMazouni, 2014).

Las técnicas de prevención y eliminación del fouling para cultivos de moluscos bivalvos se limitan a la limpieza periódica, ya sea de forma manual o bien con la aplicación de químicos como el uso de pinturas tóxicas, conocidas como antifouling, tratamientos de emersión y secado, así como control biológico. Para el biocontrol se han propuesto organismos omnívoros y herbívoros coexistiendo en las cestas de cultivo de los bivalvos; entre los organismos utilizados están crustáceos como el cangrejo de roca Cancer irroratus y el camarón de roca Rhynchocinetes typus (Hidu, Conary \& Chapman, 1981; Dumont, Urriago, Abarca, Gaymer \& Thiel, 2009), gasterópodos como Littorina littorea en cultivos de ostras (Enright et al., 1983; Cigarría, Fernández \& Magadán, 1998) y equinodermos; en éstos últimos los erizos de mar han sido recomendados para el control biológico del fouling en cultivo de ostras perlas y pectínidos (Lodeiros \& García, 2004; Ross, Thorpe \& Brand, 2004), siendo utilizados en recientes estudios (Cortés-Useche, 
Gómez-León \& Santos-Acevedo, 2011, Zhanhui et al., 2014) y empleándolos además como biocontrol de depredadores (Malavé et al., 2012). Adicionalmente, dada la demanda de los erizos de mar para el consumo humano, existe un interés adicional de considerarlos como posibles especies complementarias en policultivos con moluscos.

Una de las especies de moluscos bivalvos más promisorias para la diversificación de la acuicultura en Ecuador es la ostra del Pacífico Crassostrea gigas y se ha promovido su cultivo, en sistemas suspendidos (long lines), principalmente a través de las comunas pesqueras de la región costera central del Ecuador (Lodeiros et al. 2017). Adicionalmente, el cultivo de esta especie en cestas ubicadas en canales reservorios asociados al cultivo de camarones o en sistemas de líneas flotantes en el mar han sido también satisfactorio (Lombeida, 1997), aunque persiste el problema relacionado con el depósito de material inerte y crecimiento rápido de organismos coloniales que obstruyen a las unidades de cultivo, además de la alta incidencia de depredación por peces.

Dado el interés para el desarrollo del cultivo de la ostra $C$. gigas en Ecuador, y el problema del fouling como un factor negativo en el cultivo, en el presente estudio se evaluó el uso de dos especies de erizos de mar nativas como agentes biocontroladores del fouling en el cultivo de $C$. gigas en la provincia de Santa Elena, Ecuador.

\section{MATERIALES Y MÉTODOS}

Colecta, mantenimiento y selección de animales: Se colectaron Arbacia incisa (erizo negro) y Eucidaris thouarsii (erizo lápiz) en el litoral costero de El Palmar y Ayangue, Santa Elena, Ecuador, y fueron mantenidos con flujo continuo de agua de mar y aireación en tanques rectangulares de fibra de vidrio de $1-\mathrm{m}^{3}$ base cóncava $(22 \mathrm{~cm} \times 14 \mathrm{~cm} \times 6 \mathrm{~cm})$ en el CENAIM-ESPOL, San Pedro de Manglaralto, Santa Elena. Las ostras, provenientes de cultivos en suspensión en la Bahía de Ayangue, Provincia de Santa Elena, Ecuador, se les extrajo físicamente el fouling adherido a la concha, manteniéndose por cuatro días en tanques similares a los utilizados para los erizos, pero con una dieta de Isochrysis galbana y Chaetoceros gracilis a razón 1:1 (170000 cél.ml ${ }^{-1}$ día $\left.^{-1}\right)$. Tanto los erizos como las ostras fueron seleccionados en función de obtener lotes homogéneos, en longitudes y pesos. La talla inicial de los erizos lápiz (E. thouarsii) y negro (A. incisa) fue de $34.2 \pm 2.13 \mathrm{~mm}$ y $50.5 \pm 0.43 \mathrm{~mm}$ en diámetro de la testa (DT) y pesaron $42.5 \pm 2.51$ g y $60.8 \pm 2.60 \mathrm{~g}$, respectivamente. Las ostras (C. gigas) midieron $68.2 \pm$ $3.81 \mathrm{~mm}$ en longitud antero-posterior y pesaron $0.7 \pm 0.22$ g. La selección del bivalvo a dichas tallas fue debido a que los mismos se encuentran cercanos a la talla comercial sugerida para Ecuador $(80 \mathrm{~mm})$.

Diseño del experimento: El experimento consistió en contrastar el crecimiento y la supervivencia de $C$. gigas en condición de cultivo suspendido durante un mes, utilizando cestas japonesas o pearl nets (10 individuos por cesta) en una línea de cultivo en la Ensenada de Ayangue (1 $\left.{ }^{\circ} 59^{\prime} 1.59^{\prime \prime} \mathrm{S} ; 80^{\circ} 45^{\prime} 35.15^{\prime \prime} \mathrm{W}\right)$ con cuatro tratamientos: 1) ostras sin erizos 2) ostras con 1 erizo lápiz, 3) ostras con 1 erizo negro y 4) ostras sin erizos y con un saco protector (Fig. 1). Cada tratamiento consistió de tres cestas (tres réplicas) unidos a $20 \mathrm{~cm}$ entre ellos. El tratamiento de saco protector con malla se estableció con el objetivo de medir la cantidad de fouling en las cestas sin erizos, ya que, en el cultivo tradicional de $C$. gigas en Ecuador, los elementos de cultivo (cestas japonesas-pearl nets y linternas-lanter nets) se recubren con un saco hecho con redes tipo anchovetera (malla de 1/2") en función de proteger a las ostras de peces depredadores, particularmente de la familia Balistidae).

Fouling acumulado: Para evaluar el efecto de biocontrol del fouling de los erizos, al principio y al final del bioensayo, las cestas fueron deshidratadas en estufa a $60^{\circ} \mathrm{C}$ por $48 \mathrm{~h}$ y pesadas, de igual modo para evaluar la efectividad del uso de los erizos en el crecimiento 


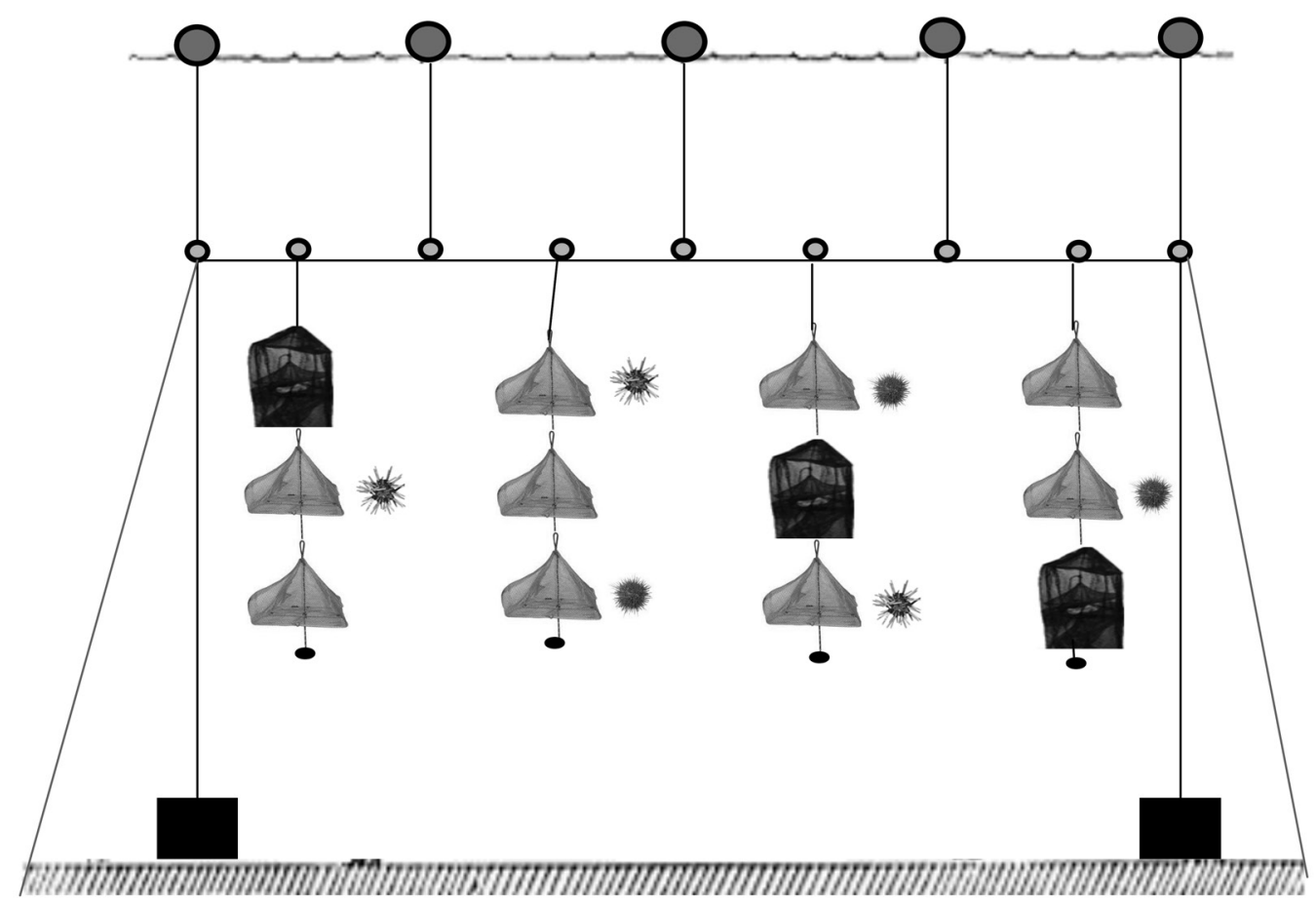

Fig. 1. Diseño experimental para evaluar el uso de los erizos Eucidaris thouarsii y Arbacia incisa como biocontrol de fouling en cestas japonesas utilizadas para el cultivo suspendido de la ostra Crassostrea gigas.

Fig. 1. Experimental design to evaluate the use of the sea urchins Eucidaris thouarsii and Arbacia incisa as fouling biocontrol in the pearl nets used for the suspended culture of the oyster Crassostrea gigas.

de C. gigas, las ostras de todas las réplicas fueron medidas en cuanto a la masa seca de sus tejidos (tratamiento a deshidratación a $60^{\circ} \mathrm{C}$ por $\left.48 \mathrm{~h}\right)$.

Análisis estadísticos: La diferencia entre tratamientos se estableció usando una prueba Anova de una vía con análisis a posteriori de Duncan. La probabilidad fijada para estas pruebas fue $\mathrm{P}=0.05$. Se determinó la normalidad de la data según la prueba $\mathrm{W}$ de Shapiro-Wilks y se verificó la homogeneidad de la varianza examinando las gráficas de distribución de residuos, siguiendo las recomendaciones en Zar (2010).

\section{RESULTADOS}

Fouling acumulado: La mayor cantidad de fouling se registró en las cestas sin erizos. En este tratamiento se acumuló la cantidad de hasta $55.3 \pm 5.80 \mathrm{~g}$ (Fig. 2). Las menores cantidades de fouling se registraron en los tratamientos con saco protector sin erizo (35.6 \pm 5.17 g, Fig. 2) y con erizo lápiz (39.1 \pm 1.91 g). No hubo diferencias significativas entre ambos tratamientos y erizo lápiz. El tratamiento de las cestas con erizo negro acumularon fouling hasta $46,1 \pm 2,84 \mathrm{~g}$.

Crecimiento de ostras: Al final del experimento no se registró ninguna mortalidad en las ostras. El mayor crecimiento lo registraron las ostras del tratamiento con erizo lápiz ( 0.49 \pm 0.089 g, Fig. 2). Este crecimiento fue significativamente mayor que el registrado en los otros tratamientos. Por otro lado, el crecimiento de las ostras del tratamiento de cestas sin erizos $(0.41 \pm 0.25$ g, Fig. 3) fue significativamente mayor que el de las ostras en las cestas con saco protector $(0.28 \pm 0.072$ g, Fig. 3$)$ y con el erizo negro $(0.27 \pm 0.139$ g, Fig. 3$)$. 


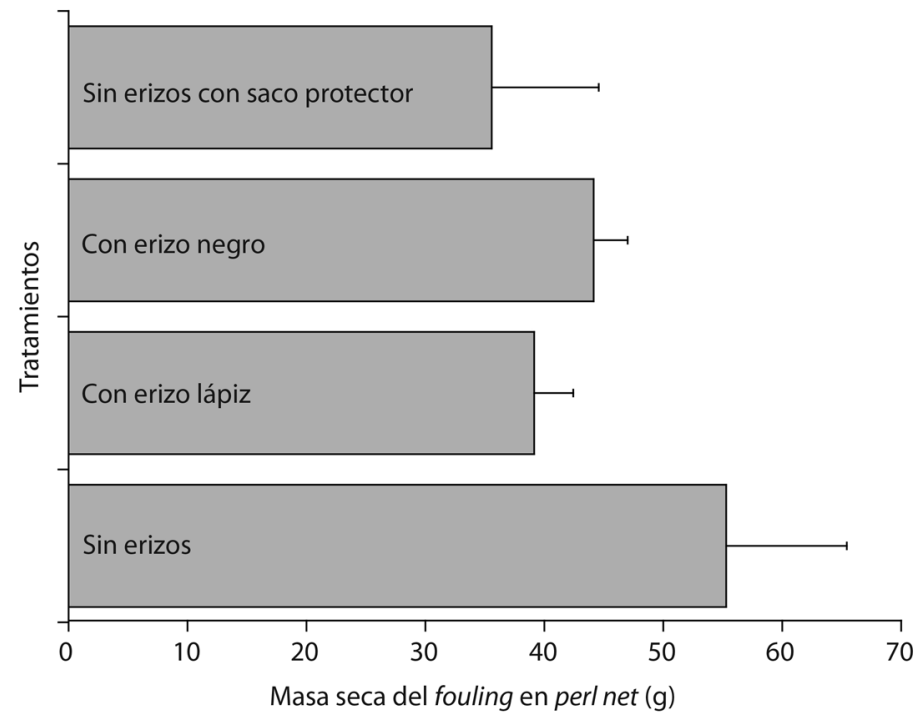

Fig. 2. Masa seca del fouling acumulado en las mallas de las cestas japonesas durante 30 días en los diferentes tratamientos. Las líneas delgadas horizontales muestran el error estándar.

Fig. 2. Dry mass of the fouling accumulated in the pearl nets during 30 days in the different treatments. The horizontal thin lines show the standard error.

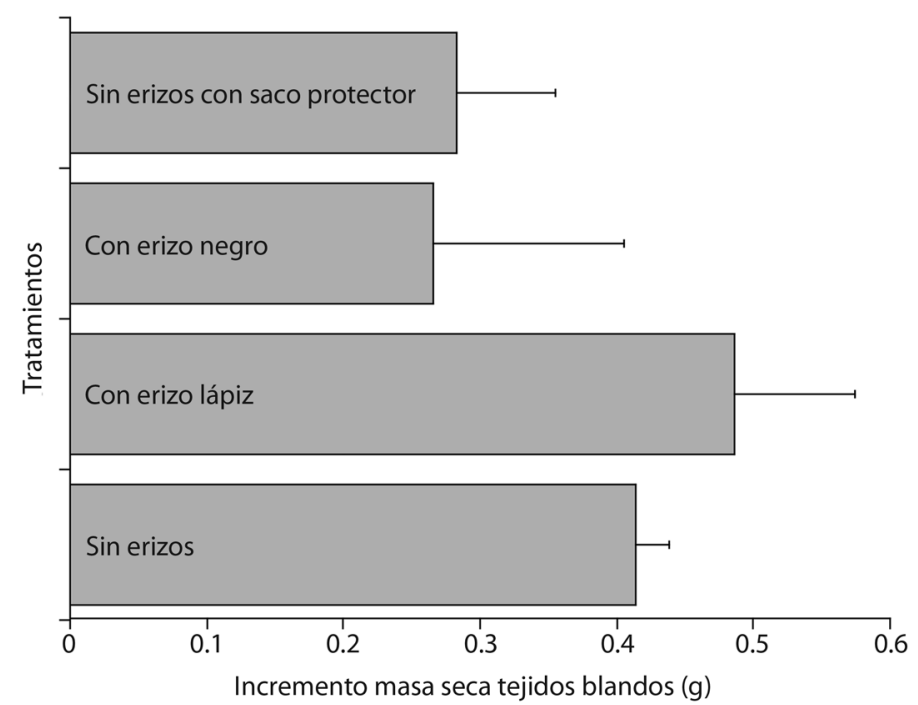

Fig. 3. Incremento de la masa seca del tejido blando de la ostra Crassostrea gigas cultivada en suspensión durante 30 días en los diferentes tratamientos. Las líneas delgadas horizontales muestran el error estándar.

Fig. 3. Increase of dry mass of the soft tissue in the oyster Crassostrea gigas cultured in suspension for 30 days in the different treatments. The horizontal thin lines show the standard error. 


\section{DISCUSIÓN}

El crecimiento del fouling en las estructuras de cultivo en la Bahía de Ayangue, es un factor importante a tener en cuenta. En tan sólo un mes de cultivo las cestas con ostras y sin erizo acumularon en masa seca unos $55 \mathrm{~g}$ de fouling, esto corresponde a aproximadamente $1 / 2 \mathrm{~kg}$ de masa húmeda de organismos y material acumulado, suponiendo una cantidad relevante de fouling en las cestas de cultivo. Ello muestra que el crecimiento de los organismos que componen el fouling, es acelerado, como ha sido encontrado en otras zonas tropicales (Lodeiros \& Himmelman, 1996; Lodeiros et al., 1998).

En este estudio no se determinó el tipo del fouling presente en las cestas; sin embargo, la biota más representativa fueron principalmente algas, briozoos, ascidias, crustáceos (Balanus spp.) y moluscos bivalvos, los cuales han sido reportados en un estudio del fouling en las cestas usadas en el cultivo de C. gigas (Alcivar, 2015), ubicado en una zona cercana (El Palmar, Provincia de Santa Elena), a unos $5 \mathrm{Km}$ del sitio de experimentación. Estos organismos forman una barrera densa que bloquea el flujo de agua al interior de las cestas haciendo evidente la limitación de la disponibilidad del alimento en el interior de las mismas. A parte de ello, los organismos filtradores que coexisten podrían competir con las ostras por el alimento disponible, creando también condiciones adversas de bajas concentraciones de oxígeno, lo cual afecta al crecimiento en moluscos bivalvos (Claereboudt, Bureau, Côté \& Himmelman, 1994; Lodeiros \& Himmelman, 1996, 2000; De Sa, Nalesso \& Paresque, 2007). Esta hipótesis se ve comprobada al observar la acción omnívora y detritívora del erizo lápiz $E$. thouarsii, que disminuyó la cantidad de fouling en las mallas en un $30 \%$, lo cual estuvo asociado al mayor crecimiento de la ostra. La acción de biocontrol del fouling en las mallas de las cestas de cultivo de moluscos bivalvos ha sido reportado para erizos de ecosistemas templados, como Psammechinus miliaris, Echinus esculentus, Hemicentrotus pulcherrimus (Ross et al., 2004, Zhanhui et al., 2014) y para el trópico con
Lytechinus variegatus y Echinometra lucunter (Lodeiros y García 2004, Cortés-Useche et al., 2011), y donde fueron observadas reducciones efectivas del fouling.

Nuestros resultados mostraron que el erizo negro $A$. incisa no ejerció un efecto importante en la disminución del fouling al interior de las cestas con ostras. Es muy probable que esta especie de erizo no pudo desarrollar su acción de "pastoreo" sobre la malla de la cesta y remover el fouling, por tener púas relativamente delgadas (comparativamente con las gruesas del erizo lápiz E. thouarsii), lo que probablemente le dificultaría su desplazamiento sobre la superficie de la cesta. Esto se evidenció mediante la presencia de púas abundantes rotas en las cestas revisadas, descartando su posible uso como biocontrol en cultivo de bivalvos usando cestas con mallas.

Las cestas con saco protector (usados tradicionalmente en algunos cultivos de C. gigas), muestran efectividad de reducir, hasta un $40 \%$ la masa del fouling, pero el crecimiento en las ostras fue el menor, junto con el tratamiento con el erizo negro $A$. incisa. Si bien, la protección permite una disminución del fouling en la cesta de cultivo (mayor superficie de fijación para los organismos del fouling), evidentemente las barreras de la malla del saco protector y el fouling que se desarrolla en él disminuyen el flujo de agua dentro de las cestas, limitando aún más el alimento que el propio fouling desarrollado en las cestas. La doble protección de malla implementada por técnicos de la Subsecretaría de Acuicultura del Ministerio de Acuicultura y Pesca (MAP) en Ecuador, en sus experiencias de cultivo de $C$. gigas en suspensión con las comunidades costeras da resultados excelentes para evitar la depredación por jaibas y peces, particularmente de la familia Balistidae, quienes condicionan la rentabilidad del cultivo (A. García, Com. Personal); no obstante, las mallas protectoras adjuntan mayor inversión, labor y mantenimiento y por ende reduce el rendimiento y rentabilidad del cultivo. Dada la disminución de disponibilidad de alimento en las cestas con doble malla, y la consecuente disminución del crecimiento en $C$. gigas, 
se hace imprescindible para la optimización del cultivo, ensayar con unidades que sean resistentes o rechacen al ataque de los depredadores.

Nuestros resultados sugieren que el fouling es un factor limitante del cultivo de la ostra Crassostrea gigas en las aguas costeras de Ecuador y que el erizo lápiz Eucidaris thouarsii puede ser utilizado como agente de biocontrol del fouling en las cestas en suspensión para el cultivo de la ostra del Pacífico Crassostrea gigas en las costas de Ecuador. Su uso como agente removedor de fouling podría no estar limitado, dado que es una especie abundante que habita en ambientes rocosos someros de la costa continental del Ecuador. No obstante, se recomienda realizar más estudios para la optimización de su uso como biocontrol.

\section{AGRADECIMIENTOS}

El trabajo se ha realizado como parte complementaria del proyecto de investigación Desarrollo de protocolo de domesticación para el uso sostenible de nuevas especies marinas para consumo de alimento y repoblación de bancos naturales, financiado por la SENESCYT. César Lodeiros participó en el estudio durante su vinculación al CENAIM-ESPOL a través del proyecto Prometeo de la SENESCYT, Ecuador.

\section{RESUMEN}

El fouling obstruye las cesta del cultivo del moluscos afectando su crecimiento. El uso de erizos de mar como biocontrolador del fouling en las redes de cestas japonesas (pearl nets) en cultivo suspendido de Crassostrea gigas se evaluó durante un mes. El diseño experimental incluyó: 1) ostras sin erizos de mar, 2) ostras con el erizo lápiz Eucidaris thouarsii (34.2 $\pm 2.13 \mathrm{~mm}$ de diámetro testa-DT), 3) ostras con el erizo negro Arbacia incisa $(50.5 \pm 0.43 \mathrm{~mm}$ DT) y 4) ostras sin erizos de mar con un saco para la protección de la cesta (saco de malla de tipo sardina utilizada para evitar la depredación de C. gigas en Ecuador). La masa seca de las cestas y de las ostras se determinaron al inicio y al final del experimento. El fouling de tratamientos sin erizos $(55.3 \pm 5,80 \mathrm{~g})$ y con A. incisa $(46.1 \pm 2.84 \mathrm{~g})$ fue elevado. Estas cantidades fueron significativamente mayores que las que se encontraron en las cestas con $E$. thouarsii $(39.1 \pm 1.9 \mathrm{~g})$ y con la malla protectora sin erizos
(35.6 $\pm 5.17 \mathrm{~g})$. El crecimiento de C. gigas con E. thouarsii fue significativamente mayor $(0.49 \pm 0.089 \mathrm{~g})$ a los otros tratamientos. Los resultados sugieren el uso del erizo $E$. thouarsii como un agente de control biológico del fouling en las mallas de cestas en el cultivo de C. gigas.

Palabras clave: redes de perlas; ostras; biocontrol; maricultura; policultivos.

\section{REFERENCIAS}

Alcivar, G. (2015). Caracterización de la macrofauna marina perjudicial, adherida en sistemas de cultivo suspendido de Crassostrea gigas, en la ensenada de Palmar, Península de Santa Elena, enero-mayo, 2015. (Tesis de Licenciatura). Universidad Estatal Península de Santa Elena, Facultad de Ciencias del Mar. pp. 134

Cigarría, J., Fernández, J., \& Magadán, L. P. (1998). Feasibility of biological control of algal fouling in intertidal oyster culture using periwinkles. Journal of Shellfish Research, 17, 1167-1169.

Claereboudt, M., Bureau, D., Côté, J., \& Himmelman, J. H. (1994). Fouling development and its effect on the growth of juvenile giant scallops (Placopecten magellanicus) in suspended culture. Aquaculture, $121,327-342$.

Cortés-Useche, C., Gómez-León, J., \& Santos-Acevedo, M. (2011). Erizos de mar como control biológico del "fouling" en un cultivo de Nodipecten nodosus en el área de Santa Marta, Caribe colombiano. Boletín de Investigaciones Marinas y Costeras, 40(2),233-247.

De Sa, F. S., Nalesso, R.C., \& Paresque K. (2007). Fouling organisms on Perna perna mussels: is it worth removing them? Brazilian Journal of Oceanography, $55,155-161$.

Dumont, C. P., Urriago, J. D., Abarca, A., Gaymer, C. F., \& Thiel, M. (2009). The native rock shrimp Rhynchocinetes typus as a biological control of fouling in suspended scallop cultures. Aquaculture, 292, 74-79.

Enright, C., Krailo, D., Staples, L., Smiht, M., Vaughan, C., Ward, D., Gaul, P., \& Borgese, E. (1984). Biological control of fouling algae in oyster aquacultureJournal of Shellfish Research, 3, 41-44.

Fitridge, I., Dempster T., Guenther J., \& De Nys R. (2012). The impact and control of biofouling in marine Aquaculture: a review. Biofouling, 28: 649-669.

Hidu, H., Conary, C., \& Chapman, S. R. (1981). Suspended culture of oysters: biological control. Aquaculture, 22, 189-192.

Lacoste, E., \& Gaertner-Mazouni, N. (2014). Biofouling impact on production and ecosystem functioning: 
a review for bivalve aquaculture. Reviews in Aquaculture, 6, 1-10.

Lodeiros, C., \& Garcia N. (2004). The use of sea urchins to control fouling during suspended culture of bivalves. Aquaculture, 231, 293-298.

Lodeiros, C., \& Himmelman, J. H. (1996). Influence of fouling on the growth and survival of the tropical scallop, Euvola (Pecten ) ziczac (L. 1758) in suspended culture. Aquaculture, Res. 27, 749-756.

Lodeiros, C., \& Himmelman, J. H. (2000). Identification of environmental factors affecting growth and survival of the tropical scallop Euvola (Pecten) ziczac in suspended culture in the Golfo de Cariaco, Venezuela. Aquaculture, 182, 91-114.

Lodeiros, C., Rodríguez-Pesantes, D., Márquez, A., Revi1la, J., \& Sonnenholzner, S. 2017. Cultivo suspendido de la ostra Crasssotrea gigas en la Bahía de Ayangue, Ecuador. Foro Ac. Rec. Mar. Rías Gal. XIX. 19: 317-330.

Lodeiros, C., Rengel, J., Freites, L., Morales, F., \& Himmelman, J. H. (1998). Comparison of growth and survival of the tropical scallop Nodipecten (Lyropecten) nodosus maintained at three depths in suspended culture. Aquaculture, 165, 41-50.
Lombeida, P. (1997). Manual para el Cultivo de Ostras en Granjas Camaroneras. Proyecto JICA-CENAIM. $25 \mathrm{pp}$.

Malavé, C., Freites, L., Lodeiros, C., Mendoza, J., Troccoli L., \& Dale A. (2012). Annual recruitment, predation rates and biocontrol of Linatella caudata (Mollusca: Gastropoda) in suspended enclosure culture of the pearl oyster Pinctada imbricata. Aquaculture, 354355, 75-83.

Ross, K., Thorpe, J. \& Brand, A. (2004). Biological control of fouling in suspended scallop cultivation. Aquaculture, 229, 99-116.

Uribe, E., Lodeiros, C., Felix-Pico E. \& Etcherepare I. (2001). Epibiontes en pectínidos de Iberoamérica. In: A.N. Maeda-Martínez (ed.), Los Moluscos Pectínidos de Iberoamérica: Ciencia y Acuicultura. Cap. 13: 249-266.

Zar, J. H., (2010). Biostatistical analysis, 5th edition. Pentice Hall, Englewoods Cliff, N.J. 944 pp.

Zhanhui, Q., Jun, W., Yuze, M., Jihong, Z., Zengjie, J., \& Jianguan F. (2014). Use of the sea urchin Hemicentrotus pulcherrimus for biological control of fouling in suspended scallop cultivation in Northern China. Aquaculture, 420, 270-274. 